Nervenarzt 2014 · 85:480

DOI 10.1007/s00115-014-4049-z

Online publiziert: 30. März 2014

(c) Springer-Verlag Berlin Heidelberg 2014

\section{C.-A. Haensch}

Klinik für Neurologie, Fakultät für Gesundheit, Krankenhaus St. Franziskus, Kliniken

Maria Hilf GmbH, Universität WittenHerdecke, Mönchengladbach

\title{
Entwicklung der Deutschen Neurologie nach 1960
}

\section{Zum Beitrag \\ Dichgans J (2013) Entwicklung der Deut- schen Neurologie nach 1960. Nervenarzt 84:1512-1522. \\ http://dx.doi.org/10.1007/s00115-013-3922-5}

In dem sehr wertvollen und lesenswerten Überblick von Dichgans über die Entwicklung der deutschen Neurologie nach 1960 findet sich leider in Tabelle 1 eine unvollständige Darstellung [1]. Seit 1994 besteht an der privaten Universität Witten/ Herdecke in der Fakultät für Gesundheit ein Lehrstuhl für Neurologie, zunächst unter dem Ordinariat von J. Jörg, in Nachfolge seit 2006 unter der Leitung von S. Isenmann. Da sich dieser Neglekt auch in anderen Übersichten findet, erscheint ein Hinweis notwendig [2]. Im Rahmen eines Modellstudienganges Humanmedizin wurden in Witten/Herdecke bereits frühzeitig aktuelle Anforderungen an die universitäre Lehre im Fach Neurologie durch das problemorientierte Lernen, „bedside teaching“ und praxisbezogene Prüfungsformen berücksichtigt [3]. Ziel ist es, eine integrative, reflektierte Medizindidaktik zu betreiben, die den Studierenden eine aktive Rolle zuweist und neben Vermittlung von Wissen und Fertigkeiten auch Wahrnehmung, neurologisches Denken und Reflexion ermöglicht [4]. Klinische Forschungsschwerpunkte des Lehrstuhls lagen bisher im Bereich des autonomen Nervensystems, des Morbus Parkinson und der Schlafmedizin.

\section{Korrespondenzadresse}

\section{Prof. Dr. C.-A. Haensch}

Klinik für Neurologie, Fakultät für Gesundheit, Krankenhaus St. Franziskus, Kliniken Maria Hilf $\mathrm{GmbH}$, Universität WittenHerdecke Viersener Str. 450, 41063 Mönchengladbach carl-albrecht.haensch@mariahilf.de

Interessenkonflikt. C.-A. Haensch gibt an, dass kein Interessenkonflikt besteht.

\section{Literatur}

1. Dichgans J (2013) Entwicklung der Deutschen Neurologie nach 1960. Nervenarzt 84(12): 1512-1522

2. N.N. (1995) Neurologische Universitätskliniken und Hochschulabteilungen. In: Elger C, Dengler R (Hrsg) Jahrbuch der Neurologie 1995. Biermann, Zülpich, S 213-236

3. Isenmann S, Biesalski A, Zupanic M, Gerloff C (2013) Universitäre Lehre in der Neurologie. Aktuelle Situation und zukünftiger Bedarf. Nervenarzt 84:1220-1227

4. Wild D, Schüle S, Rimpau W (2000) Klinische Neuroanatomie an der Universität Witten/ Herdecke. Med Ausbild 17:36-41 